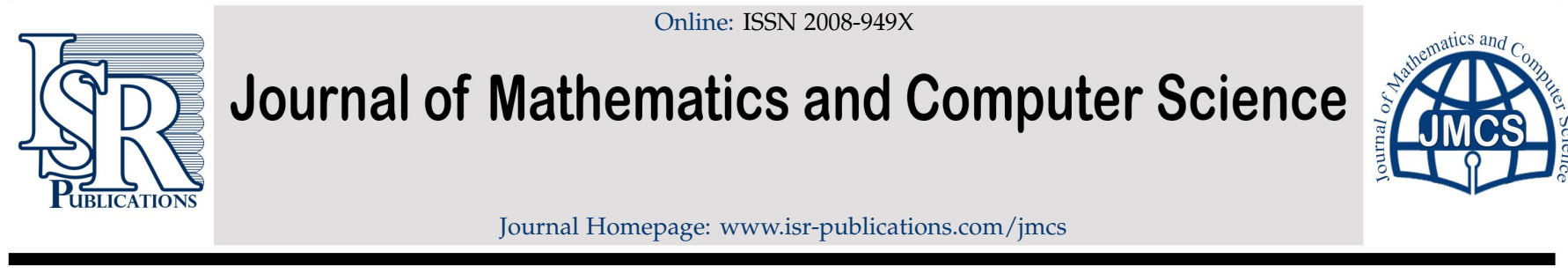

\title{
Weak prime L-fuzzy filters of semilattices
}

\author{
Ch. Santhi Sundar Raj ${ }^{\mathrm{a}}$, K. Ramanuja Rao ${ }^{\mathrm{b}, *}$, B. Subrahmanyam ${ }^{\mathrm{a}}$ \\ ${ }^{a}$ Department of Engineering Mathematics, Andhra University, Visakhapatnam, 530003, India. \\ ${ }^{b}$ Deaprtment of Mathematics, Fiji National Uniersity, Lautoka, FIJI.
}

\begin{abstract}
The concept of weak prime L-fuzzy filter of a semilattice $S$ is introduced and example are given. A characterization of weak prime L-fuzzy filters is established and prime filters of $S$ are identified with weak prime L-fuzzy filters. Also, minimal weak prime L-fuzzy filters are characterized.
\end{abstract}

Keywords: Bounded semilattice, L-fuzzy filter, prime L-fuzzy filter, weak prime L-fuzzy filter, frame.

2020 MSC: 06D72, 06F15, 08A72.

(C)2022 All rights reserved.

\section{Introduction}

Zadeh, in his pioneering work [11] introduced the notion of a fuzzy subset $A$ of a non-empty set $X$ as a function from $X$ into $[0,1]$. Rosenfield [6] applied this notion to develop the theory of groups. Goguen [1] generalized and continued the work of Zadeh and realized that the unit interval $[0,1]$ is not sufficient to take the truth values of general fuzzy statements. Therewith, several researchers took interest to the fuzzyfication of algebraic structures. In which, Kuroki [2], Liu [3], Malik and Mordersan [4], and Mukherjee and Sen [5] are engaged in fuzzifying various concepts and obtained significant results of algebras.

Further, Swamy and Swamy [10] have introduced the concept of a fuzzy prime ideal of a ring and developed the theory of fuzzy ideals by assuming truth values in a complete lattice L satisfying the infinite meet distributive law, such lattices are called frames. The concept of prime ideal is vital in the study of structure theory of distributive lattices. In [8], the authors have introduced and studied the notion of L-fuzzy filters of a semilattice $S$ with truth values in a frame $L$. It is proved that $S$ is distributive iff the lattice $\mathcal{F}(S)$ of all filters of $S$ is distributive iff the lattice $\mathcal{F}_{L}(F(S))$ of all L-fuzzy filters of $S$ is distributive. In [9], the authors have introduced the concept of prime L-fuzzy filters of a bounded semilattice $S$, which are meet-prime elements in the lattice $\mathcal{F}_{L}(\mathcal{F}(S))$. Further, in [7] the authors have introduced the notion of L-fuzzy ideals of a semilattice $S$ and obtained significant results on this.

\footnotetext{
*Corresponding author

Email addresses: santhisundarraj@yahoo.com (Ch. Santhi Sundar Raj), ramanuja.kotti@fnu.ac.fj (K. Ramanuja Rao), bollasubrahmanyam@gamil.com (B. Subrahmanyam)
}

doi: $10.22436 /$ jmcs.024.01.01 
The aim of this paper is to study the L-fuzzy filters $A$ of a bounded semilattice $S$ for which each $\alpha$-cut $A_{\alpha}$ i.e., $A_{\alpha}=\{x \in S: A(x) \geqslant \alpha\}$ is either a prime filter of $S$ or whole semilattice $S$. This paper consists of four sections. In the second section we recall some definitions and certain results. In third section we introduce the concept of a weak prime L-fuzzy filter (WPLF) of a bounded semilattice $S$ and characrerize these. Fourth section deals with minimal weak prime L-fuzzy filters (Minimal WPLFs).

Throughout this paper, $S$ stands for a bounded semilattice $(S, \wedge, 0,1)$ unless otherwise stated. And, $L$ stands for a non-trivial frame $(L, \wedge, \vee, 0,1)$; i.e., a complete lattice satisfying the infinite meet distributive law

$$
\alpha \wedge\left(\bigvee_{\beta \in T} \beta\right)=\bigvee_{\beta \in T}(\alpha \wedge \beta),
$$

for all $\alpha \in \mathrm{L}$ and any $\mathrm{T} \subseteq \mathrm{L}$. Here the operations $\wedge$ and $\vee$ are supremum and infimum in the lattice $\mathrm{L}$. An element $1 \neq c \in L$ is said to be meet-prime if, for any $a, b \in L$ and $a \wedge b \leqslant c$ imply $a \leqslant c$ or $b \leqslant c$.

\section{Preliminaries}

In this section we collect basic definitions and certain results from $[8,9]$, that we need in sequel.

A semilattice (meet-semilattice) is an algebra $S=(S, \wedge)$ satisfying the axioms

(1) $x \wedge x=x$;

(2) $x \wedge y=y \wedge x$; and

(3) $x \wedge(y \wedge z)=(x \wedge y) \wedge z$, for all $x, y, z \in S$.

If we define $x \leqslant y$ iff $x \wedge y=x$, then $\leqslant$ is a partial order on $S$ in which $x \wedge y$ is the inf $\{x, y\}$ in $S$. A non-empty subset $F$ of $S$ is said to be final segment of $S$ if, for any $x \in F, y \in S$ and $x \leqslant y$ implies $y \in F$. A filter of a semilattice $S$ is a final segment $F$ of $S$ such that $x \wedge y \in F$ for all $x, y \in F$. The principal filter generated by an element $a$ of $S$, i.e., the set $\{x \in S: x \geqslant a\}$ will be denoted by [a). A proper filter $P$ of a semilattice $S$ is said to be prime if whenever two filters $G$ and $H$ are such that $\phi \neq G \cap H \subseteq P$ imply either $\mathrm{G} \subseteq \mathrm{P}$ or $\mathrm{H} \subseteq \mathrm{P}$ (or equivalently, if, for any $\mathrm{a}, \mathrm{b}$ are such that $\mathrm{a} \notin \mathrm{P}$ and $\mathrm{b} \notin \mathrm{P}$ imply the existence of $x \in S$ such that $a \leqslant x, b \leqslant x$ and $x \notin P)$.

Definition 2.1. Let $X$ be any non-empty set and $L$ a frame. Any function $A: X \rightarrow L$ is called an L-fuzzy subset of $X$. For any L-fuzzy subset $A$ of $X$ and $\alpha \in L, A_{\alpha}$ denotes $\alpha$-cut of $A$, i.e.,

$$
A_{\alpha}=\{x \in X: \alpha \leqslant A(x)\}
$$

Definition 2.2. For any L-fuzzy subsets $A$ and $B$ of $X$, define

$$
A \leqslant B \Leftrightarrow A(x) \leqslant B(x), \text { for all } x \in X .
$$

Then $\leqslant$ is a partial order on the set of L-fuzzy subsets of $X$ and is called the point wise ordering.

Result 1. Let $A$ and $B$ be L-fuzzy subsets of $X$. Then

$$
A \leqslant B \Leftrightarrow A_{\alpha} \subseteq B_{\alpha}, \quad \text { for all } \alpha \in L
$$

Definition 2.3. A proper L-fuzzy subset $A$ of $X$ is a non-constant L-fuzzy subset of $X$, i.e., $A(x) \neq 1$ for some $x \in X$.

Definition 2.4. An L-fuzzy subset $A$ of $S$ is said to be an L-fuzzy filter of $S$ if,

$$
A\left(x_{0}\right)=1, \text { for some } x_{0} \in S,
$$

and

$$
A(x \wedge y)=A(x) \wedge A(y), \quad \text { for all } x, y \in S
$$


Result 2. The following are equivalent to each other, for any L-fuzzy subset A of S,

(1) $A$ is an L-fuzzy filter of $S$.

(2) $A\left(x_{0}\right)=1$ for some $x_{0} \in S, A(x \wedge y) \geqslant A(x) \wedge A(y)$ and $x \leqslant y \Rightarrow A(y) \geqslant A(x)$.

(3) $A_{\alpha}$ is a filter of $S$, for all $\alpha \in L$.

Result 3. Let $A$ be a fuzzy filter of $S$ and $X$ a non-empty subset of $S$, and $x, y \in S$. We have

(1) $x \in[X) \Rightarrow A(x) \geqslant \bigwedge_{i=1}^{m} A\left(a_{i}\right)$ for some $a_{1}, a_{2}, \cdots a_{m} \in X$, where

$$
[X)=\left\{a \in S: \bigwedge_{i=1}^{n} x_{i} \leqslant a \text { for some } x_{i} \in X\right\}
$$

(2) $x \in[y) \Rightarrow A(x) \geqslant A(y)$.

(3) If $S$ is bounded then $A(0)<1$ and $A(1)=1$.

Result 4. Let $(S, \wedge)$ be a bounded semilattice and $\mathcal{F}_{L}(F(S))$ denote the lattice all L-fuzzy filters of $S$. Then the following are equivalent to each other:

(1) $\mathcal{F}_{\mathrm{L}}(\mathcal{F}(\mathrm{S}))$ is a distributive.

(2) $F(S)$ is a distributive, where $F(S)$ denotes the lattice of filters of $S$.

(3) $S$ is distributive.

Definition 2.5. A proper L-fuzzy filter $A$ of a bounded semilattice $S$ is said to be prime L-fuzzy filter of $S$ if, for any L-fuzzy filters B and $C$ of $S$,

$$
B \wedge C \leqslant A \Rightarrow B \leqslant A \text { or } C \leqslant A,
$$

where $(B \wedge C)(x)=B(x) \wedge C(x)$.

Result 5. Let $A$ be an L-fuzzy fileter of $S$. Then $A$ is prime L-fuzzy filter of $S$ if and only if, the following are satisfied.

(1) $|\operatorname{Im}(A)|=2$, i.e., $A$ is two-valued.

(2) For any $x \in S$, either $A(x)=1$ or $A(x)$ is meet-prime element in $L$.

(3) $A_{1}$ is a prime filter of $S$.

Result 6. Let $A$ be an L-fuzzy filter of $S$. Then $A$ is a prime L-fuzzy filter of $S$ iff there exists a prime filter $P$ of $S$ and a meet-prime element $\alpha$ in L such that $A=A_{\alpha}^{P}$, where

$$
A_{\alpha}^{P}(x)= \begin{cases}1 & \text { if } x \in P, \\ \alpha & \text { if } x \notin P .\end{cases}
$$

\section{Weak prime L-Fuzzy filters (WPLF)}

Let us recall that an L-fuzzy subset $A$ of $S$ is an L-fuzzy filter of $S$ iff $A_{\alpha}$ is a filter of $S$ for each $\alpha \in L$.

Definition 3.1. A proper L-fuzzy filter $A$ of $S$ is called a weak prime L-fuzzy filter (WPLF), if for each $\alpha \in L, A_{\alpha}$ is a prime filter of $S$ or $A_{\alpha}=S$. 
Example 3.2. Consider the semilattice $S$ whose Hasse-diagram is as depicted in Figure 1 and $L=[0,1]$, the closed interval of real numbers which is a frame in which, for any $x, y \in L$,

$$
x \vee y=\max \{x, y\}, x \wedge y=\min \{x, y\}
$$

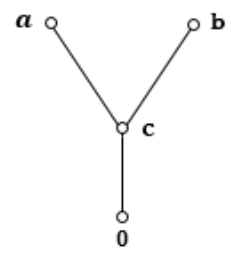

Figure 1: Hasse-diagram of Semilattice $S$.

Clearly $\{a\},\{b\}$ and $\{a, b, c\}$ are all prime filters of $S$. Now, define $A: S \rightarrow L$ as follows:

$$
A=\{(0,0),(c, 0.5),(b, 0.5),(a, 1)\} .
$$

Then $A$ is a WPLF; since, the $\alpha$-cuts of $A$ are

$$
\begin{aligned}
A_{0} & =S, \\
A_{1} & =\{a\}, \\
A_{0.5} & =\{a, b, c\}, \\
A_{\alpha} & =\{a\}, \quad \text { for any } \alpha \in(0.5,1),
\end{aligned}
$$

and

$$
A_{\alpha}=\{a, b, c\}, \quad \text { for any } \alpha \in(0,0.5) \text {. }
$$

Theorem 3.3. Let $\mathrm{A}$ be a proper $\mathrm{L}-$ fuzzy filter of $\mathrm{S}$. If $\mathrm{A}$ is a WPLF of $\mathrm{S}$, then $\operatorname{Im}(\mathrm{A})$ is a chain.

Proof. Let $a$ and $b \in S$ and put $\alpha=A(a) \vee A(b)$. Then,

$$
\begin{aligned}
x \in[a) \cap[b] & \Rightarrow a \leqslant x \text { and } b \leqslant x \\
& \Rightarrow A(a) \leqslant A(x) \text { and } A(b) \leqslant A(x) \\
& \Rightarrow \alpha=A(a) \vee A(b) \leqslant A(x) \\
& \Rightarrow x \in A_{\alpha} .
\end{aligned}
$$

Therefore $[a) \cap[b] \subseteq A_{\alpha}$. Since $A_{\alpha}$ is prime, $[a] \subseteq A_{\alpha}$ or $[b] \subseteq A_{\alpha}$.

$$
\begin{aligned}
{[a) \subseteq A_{\alpha} \Rightarrow a \in A_{\alpha} } & \Rightarrow \alpha=A(a) \vee A(b) \leqslant A(a) \\
& \Rightarrow A(b) \leqslant A(a) .
\end{aligned}
$$

Similarly, $[b) \subseteq A_{\alpha} \Rightarrow A(a) \leqslant A(b)$. Thus $\operatorname{Im}(A)$ is a chain in L.

The converse of above theorem is not true. For, consider the following example.

Example 3.4. Consider two lattices $S$ and $\mathrm{L}$ whose Hasse-diagrams are given in Figure 2 and Figure 3 respectively, where $S=\{0, c, a, b, 1\}$ and $L=\{0, s, 1\}$. 


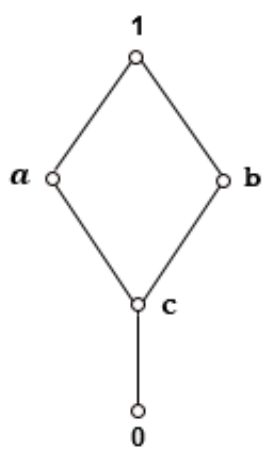

Figure 2: Hasse-diagram of lattice $S$.

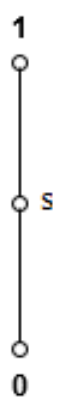

Figure 3: Hasse-diagram of lattice L.

Clearly for any L-fuzzy filter $A$ of $S, \operatorname{Im}(A)$ is a chain. Define $A: S \rightarrow L$ as

$$
A=\{(0,0),(c, s),(a, s),(b, s),(1,1)\} .
$$

Then the $\alpha$-cuts of $A$ are $A_{0}=S, A_{s}=\{c, a, b, 1\}$ and $A_{1}=\{1\}$, which are filters of $S$. Therefore $A$ is an L-fuzzy filter of $S$. However $A$ is not WPLF because $A_{1}$ is not prime since $[a) \cap[b]=\{1\}$.

The following gives a characterization of WPLFs.

Theorem 3.5. For any L-fuzzy filter A of S, the following are equivalent:

(1) A is a WPLF of $\mathrm{S}$.

(2) For any $\mathrm{a}$ and $\mathrm{b} \in \mathrm{S}$,

$$
\bigwedge\{A(x): x \in[a) \cap[b)\}=A(a) \text { or } A(b) .
$$

(3) For any $\mathrm{a}$ and $\mathrm{b} \in \mathrm{S}$,

$$
\bigwedge\{A(x): x \in[a) \cap[b)\}=A(a) \vee A(b)
$$

and

$$
\operatorname{Im}(A) \text { is a chain in } L .
$$

Proof. First note that for any $a$ and $b \in S$,

$$
A(a) \text { and } A(b) \leqslant \bigwedge\{A(x): x \in[a) \cap[b)\} .
$$

$(1) \Rightarrow(2):$ Let $a$ and $b \in S$ and put $\alpha=\bigwedge\{A(x): x \in[a) \cap[b)\}$. Then $\alpha \leqslant A(x)$ for all $x \in[a) \cap[b)$, so that $[a) \cap[b] \subseteq A_{\alpha}$. By (1) $A_{\alpha}$ is a prime filter of $S$ and hence $[a] \subseteq A_{\alpha}$ or $[b] \subseteq A_{\alpha}$. So that $a \in A_{\alpha}$ or $b \in A_{\alpha}$, i.e., $\alpha \leqslant A(a)$ or $\alpha \leqslant A(b)$. This implise $\alpha=A(a)$ or $A(b)$.

(2) $\Rightarrow$ (3): Let $a$ and $b \in S$. Then, by (2),

$$
\bigwedge\{A(x): x \in[a) \cap[b)\}=A(a) \text { or } A(b),
$$

and hence $A(b) \leqslant A(a)$ or $A(a) \leqslant A(b)$. Therefore $\operatorname{Im}(A)$ is a chain in L. Also, by (2) and since $A(a), A(b)$ are lower bounds of $\{A(x): x \in[a) \cap[b)\}$, it follows that

$$
\bigwedge\{A(x): x \in[a) \cap[b)\}=\max \{A(a), A(b)\}=A(a) \vee A(b) .
$$


(3) $\Rightarrow(1)$ : Let $\alpha \in$ L. Such that $A_{\alpha} \neq S$. Let $G$ and $H$ be two filters of $S$ such that $G \nsubseteq A_{\alpha}$ and $H \nsubseteq A_{\alpha}$. Then, $\alpha \not \leq A(a)$ and $\alpha \not \leq A(b)$ for some $a, b \in S$. By (3), $A(a) \leqslant A(b)$ or $A(b) \leqslant A(a)$. Hence,

$$
\alpha \not \leq \max \{A(a), A(b)\}=A(a) \vee A(b) .
$$

Also, by (3),

$$
\alpha \not \leq \bigwedge A(x): x \in[a) \cap[b)\} .
$$

Hence $\alpha \not \leq A(x)$ for some $x \in[a) \cap[b)$. This implise $G \cap H \nsubseteq A_{\alpha}$. Hence $A_{\alpha}$ is a prime filter of $S$. Thus $A$ is a WPLF of $S$.

Now, we slightly generalize an $\alpha$-level L-fuzzy filter $A_{\alpha}^{F}$ corresponding to a filter $F$ (see Result 6).

Definition 3.6. For any filter $F$ of $S$ and $\alpha, \beta \in L$, define an L-fuzzy subset $A_{\alpha, \beta}^{F}$ of $S$ as follows:

$$
A_{\alpha, \beta}^{F}(x)= \begin{cases}1 & \text { if } x=1 \\ \alpha & \text { if } 1 \neq x \in F \\ \beta & \text { if } x \notin F .\end{cases}
$$

Note that $A_{1, \beta}^{F}=A_{\beta}^{F}$ and $A_{1,0}^{F}=\chi_{F}$, the characteristic function corresponding to $F$.

The following is straight forward verification.

Lemma 3.7. Let $\mathrm{F}$ be a proper filter of $\mathrm{S}$ and $\alpha, \beta \in \mathrm{L}$. Then

$$
A_{\alpha, \beta}^{\mathrm{F}} \text { is an L-fuzzy filter of } S \text { iff } \beta \leqslant \alpha,
$$

and, in the case,

$$
A_{\alpha, \beta}^{F} \text { is proper iff } \beta<1 \text {. }
$$

Theorem 3.8. For any proper filter $\mathrm{P}$ of $\mathrm{S}$, the following are equivalent:

(1) $\mathrm{P}$ is a prime filter of $\mathrm{S}$.

(2) $A_{1, \beta}^{P}$ is a WPLF of $S$ for each $\beta<1$.

(3) $\chi_{\mathrm{P}}$ is a WPLF of $\mathrm{S}$.

Proof. $(1) \Rightarrow(2)$ : Suppose $P$ is prime and let $\beta<1$ in L. Put $A=A_{1, \beta}^{P}$. Then,

$$
A(x)= \begin{cases}1 & \text { if } x \in P, \\ \beta & \text { if } x \notin P .\end{cases}
$$

Let $a$ and $b \in S$. Then,

$$
\begin{aligned}
a \in P \text { or } b \in P & \Rightarrow A(a)=1 \text { or } A(b)=1 \text { and }[a) \cap[b) \subseteq P \\
& \Rightarrow A(a)=1 \text { or } A(b)=1 \text { and } A(x)=1 \text { for all } x \in[a) \cap[b) \\
& \Rightarrow \bigwedge\{A(x): x \in[a) \cap[b)\}=1=A(a) \text { or } A(b) . \\
a \notin P \text { and } b \notin P & \Rightarrow A(a)=\beta=A(b) \text { and }[a) \cap[b) \nsubseteq P \\
& \Rightarrow A(a)=\beta=A(b) \text { and } x \notin P \text { for some } x \in[a) \cap[b) \\
& \Rightarrow \bigwedge\{A(x): x \in[a) \cap[b)\}=\beta=A(a)=A(b)
\end{aligned}
$$


Therefore

$$
\bigwedge\{A(x): x \in[a) \cap[b)\}=A(a) \text { or } A(b) .
$$

Thus $A$ is WPLF.

(2) $\Rightarrow(3)$ : It is clear by the fact that $\chi_{P}=A_{1,0}^{P}$.

$(3) \Rightarrow(1)$ : Suppose $\chi_{P}$ is WPLF. Let $a$ and $b \in S$ such that $a \notin P$ and $b \notin P$. Then $\chi_{P}(a)=0=\chi_{P}(b)$.

By supposition and hence by Theorem 3.5,

$$
\bigwedge\left\{\chi_{P}(x): x \in[a) \cap[b)\right\}=\chi_{P}(a) \text { or } \chi_{P}(b) .
$$

So that

$$
\bigwedge\left\{x_{\mathrm{P}}(x): x \in[a) \cap[b)\right\}=0 .
$$

Hence $\chi_{P}(x)=0$ for some $x \in[a) \cap[b)$. (for, $\chi_{P}(x)=1$ for all $x \in[a) \cap[b) \Rightarrow \chi_{P}(a)=1$ or $\chi_{P}(b)=1$; $a$ contradiction). Therefore $x \notin P$. So that $[a) \cap[b] \nsubseteq P$. Thus $P$ is prime.

Lemma 3.9. For any bounded semilattice $S$, the following are equivalent:

(1) [1) is a meet-prime element in the lattice $\mathcal{F}(S)$ of all filters of $S$.

(2) For any $1 \neq \mathrm{a}$ and $1 \neq \mathrm{b} \in \mathrm{S}$, there exists $1 \neq \mathrm{c} \in \mathrm{S}$ such that $\mathrm{c} \geqslant \mathrm{a}$ and $\mathrm{b}$, i.e., $\mathrm{c} \in[\mathrm{a}) \cap[\mathrm{b})$.

Theorem 3.10. Le $\mathrm{P}$ be a proper filter of $\mathrm{S}$ and suppose that $[1)$ is a meet-prime element in the lattice $\mathcal{F}(\mathrm{S})$ of filters of $\mathrm{S}$. Then $\mathrm{P}$ is prime iff $\mathrm{A}_{\alpha, \beta}^{\mathrm{P}}$ is WPLF for all $1 \neq \beta \leqslant \alpha$ in $\mathrm{L}$.

Proof. Suppose $\mathrm{P}$ is prime and $1 \neq \beta \leqslant \alpha \in \mathrm{L}$. Put $A=A_{\alpha, \beta}^{\mathrm{P}}$. Then $A$ is a proper L-fuzzy filter of $S$ (by Lemma 3.7). Let $a$ and $b \in S$. Then $A(a)$ and $A(b) \leqslant A(x)$ for all $x \in[a) \cap[b)$. Let $\gamma \in$ L such that $\gamma \leqslant A(x)$ for all $x \in[a) \cap[b)$. Now,

$$
\begin{aligned}
a=1 \text { or } b=1 & \Rightarrow A(a)=1 \text { or } A(b) \text { and hence } V \leqslant A(a)=1 \text { or } A(b) \\
& \Rightarrow \bigwedge\{A(x): x \in[a) \cap[b)\}=A(a) \text { or } A(b) \\
a \notin P \text { and } b \notin P & \Rightarrow A(a)=\beta=A(b) \text { and }[a) \cap[b) \not P \\
& \Rightarrow A(a)=\beta=A(b) \text { and } A(x)=\beta \text { for some } x \in[a) \cap[b) \\
& \Rightarrow \gamma \leqslant A(x)=\beta=A(a)=A(b) \\
& \Rightarrow \bigwedge\{A(x): x \in[a) \cap[b)\}=A(a)=A(b)
\end{aligned}
$$

and

$$
\begin{aligned}
1 \neq a \in P, 1 \neq b \in P & \Rightarrow A(a)=\alpha=A(b) \text { and there exsits } 1 \neq c \in S \\
& \text { suchthat } c \in[a) \cap[b) \subseteq P \\
& \Rightarrow \gamma \leqslant A(c)=\alpha=A(a)=A(b) \\
& \Rightarrow \bigwedge\{A(x): x \in[a) \cap[b)\}=A(a)=A(b) .
\end{aligned}
$$

Thus, by Theorem 3.5, $A$ is WPLF.

Finally in this section we discuss an inter-relationship between prime L-fuzzy filters (refer Result 6) and WPLFs.

Theorem 3.11. Every prime L-fuzzy filter of $S$ is WPLF.

Proof. Let B be a Prime L-fuzzy filter of $S$. Then, $B=A_{\alpha}^{P}$ for some prime filter $\mathrm{P}$ of $S$ and a meet-prime element $\alpha$ in L. Since $P$ is prime and $\alpha<1$, we have $A_{\alpha}^{P}$ is a WPLF of $S$ (by Theorem 3.8). Thus B is WPLF. 
The converse of the above theorem is true. For, consider the example given in the following.

Example 3.12. Let $S$ be the 5-element lattice $\{0, b, c, a, 1\}$ represented by the Hasse-diagram given below Figure 4 and $\mathrm{L}$ be the 3 -element chain $\{0, s, 1\}$ with $0<s<1$.

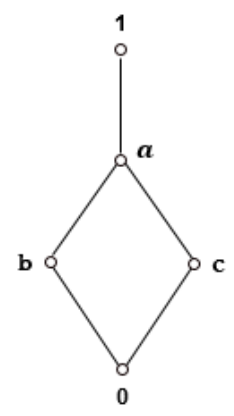

Figure 4: Hasse-diagram of 5-element lattice $S$.

Define $A: S \rightarrow L$ by $A=\{(0,0),(b, s),(c, 0),(a, s),(1,1)\}$. Then $A$ is a proper $L-f u z z y$ filter of $S$. Here the $\alpha$-cuts of $A$ are $A_{0}=S, A_{s}=\{b, a, 1\}$ and $A_{1}=\{1\}$, which are prime filters of $S$. Hence $A$ is WPLF. But $A$ is not Prime L-fuzzy filter since $A$ is not two-valued.

\section{Minimal WPLF}

By a minimal prime filter $M$ of $S$, we mean that there is no prime filter $Q$ of $S$ such that $Q \subset M$ and analogously, a minimal WPLF is a minimal element in the set of all WPLFs under the point-wise partial ordering.

Theorem 4.1. Let $\mathrm{A}$ be a WPLF of $\mathrm{S}$. If $\mathrm{A}$ is a minimal WPLF of $\mathrm{S}$, then $\mathrm{A}_{1}$, i.e., 1-cut of $\mathrm{A}$ is a minimal prime filter of $\mathrm{S}$.

Proof. Suppose that $A$ is a minimal WPLF of $S$. Then $A_{1}=\{x \in S: A(x)=1\}$ is a prime filter of $S$. To prove $A_{1}$ is minimal, let $Q$ be a prime ideal of $S$ such that $Q \subset A_{1}$. Then, choose $x \in A_{1}$ such that $x \notin Q$. Since $Q$ is prime and hence by Theorem 3.8, $x_{Q}$ is a WPLF of $S$ and $x_{Q}(x)<A(x)$. Therefore $\chi_{Q} \supsetneqq A$. This shows that $A$ is not minimal; a contradiction. Thus $A_{1}$ is a minimal prime filter of $S$.

Converse of above theorem is not true. For example, in Example 3.2, $A$ is an WPLF and $A_{1}=$ $\{a\}$ which is a minimal prime filter of $S$. But $A$ is not minimal. If we define $B: S \rightarrow L$ by $B=$ $\{(0,0),(c, 0.25),(b, 0.25),(a, 1)\}$, then $B$ is a WPLF of $S$ and $B \supsetneqq A$.

Theorem 4.2. Let $\mathrm{A}$ be a WPLF of $\mathrm{S}$ and $[1)$ is a meet-prime element in the lattice $\mathcal{F}(\mathrm{S})$ filters of $\mathrm{S}$. Then, $\mathrm{A}$ is a minimal WPLF of $\mathrm{S}$ iff, $\mathrm{A}_{\alpha}$ is a minimal prime filter of $\mathrm{S}$, for each $\alpha \in \mathrm{L}$.

Proof. Assume that $A$ is a minimal WPLF of $S$. If $A_{\beta}$ is not a minimal prime filter of $S$ for some $0<\beta<1$. Then, there exists a prime filter $P$ of $S$ such that $P \subset A_{\beta}$. Now, define $B: S \rightarrow$ L by

$$
B(x)= \begin{cases}1 & \text { if } x=1 \\ \beta & \text { if } 1 \neq x \in P \\ 0 & \text { if } x \notin P .\end{cases}
$$

Clearly $B=A_{\beta, 0}^{P}$. By Theorem 3.5, $B$ is a WPLF of $S$. As $P \subset A_{\beta}$, choose $y \in A_{\beta}$ such that $y \notin P$. Then,

$$
\beta \leqslant A(y) \text {, and } B(y)<A(y) .
$$


Also $B(x) \leqslant A(x)$ for all $x \neq y \in S$. Therefore $B \supsetneqq A$; a contradiction to our assumption. Thus $A_{\alpha}$ is a minimal prime filter of $S$ for all $\alpha \in \mathrm{L}$.

Conversely, assume that $A_{\alpha}$ is a minimal prime filter of $S$ for all $\alpha \in$ L. If B is a WPLF of $S$ such that $\mathrm{B} \leqslant \mathrm{A}$. Then, $\mathrm{B}_{\alpha} \subseteq \mathrm{A}_{\alpha}$ for all $\alpha \in \mathrm{L}$. By assumption, $\mathrm{A}_{\alpha}=\mathrm{B}_{\alpha}$ for all $\alpha \in$ L. Hence $\mathrm{B}=\mathrm{A}$. Thus $\mathrm{A}$ is a minimal WPLF of $S$.

\section{Acknowledgment}

The authors thanks the editor and anonymous reviewers for their careful reading and useful comments that have resulted in a significant improvement of the manuscript.

\section{References}

[1] J. A. Goguen, L-fuzzy sets, J. Math. Anal. Appl., 18 (1967), 145-174. 1

[2] N. Kuroki, On fuzzy ideals and fuzzy bi-ideals in semigroups, Fuzzy Sets and Systems, 5 (1981), 203-215. 1

[3] W. J. Liu, Fuzzy invariant subgroups and fuzzy ideals, Fuzzy Sets and Systems, 8 (1982), 133-139. 1

[4] D. S. Malik, J. N. Mordeson, Extensions of fuzzy subrings and fuzzy ideals, Fuzzy Sets Systems, 45 (1992), $245-251.1$

[5] T. K. Mukherjee, M. K. Sen, On fuzzy ideals of a ring I, Fuzzy Sets and Systems, 21 (1987), 99-104. 1

[6] A. Rosenfeld, Fuzzy groups, J. Math. Anal. Appl., 35 (1971), 512-517. 1

[7] Ch. Santhi Sundar Raj, B. Subrahmanyam, G. Sujatha, S. Nageswara Rao, L-fuzzy ideals of Semilattices, Int. J. Math. Trends Tech., 66 (2020), 160-175. 1

[8] Ch. Santhi Sundar Raj, B. Subrahmanyam, U. M. Swamy, Fuzzy Filters of Meet-Semilattices, Int. J. Math. Appl., 7 (2019), 67-76. 1, 2

[9] Ch. Santhi Sundar Raj, B. Subrahmanyam, U. M. Swamy, Prime L-fuzzy filters of a Semilattice, Ann. Fuzzy Math. Inform., 20 (2020), 79-87. 1, 2

[10] U. M. Swamy, K. L. N. Swamy, Fuzzy Prime Ideals of Rings, J. Math. Anal. Appl., 134 (1988), 94-103. 1

[11] L. A. Zadeh, Fuzzy sets, Inform. Control, 8 (1965), 338-353. 1 